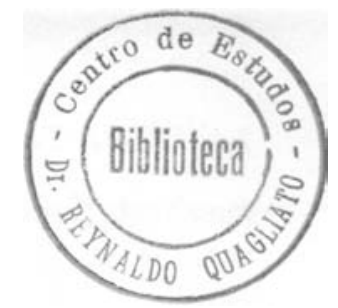

\title{
Dissociation between allergy and immunity in mycobacterial infections
}

\section{J L TURK}

Department of Pathology, Royal College of Surgeons of England, Lincoln's Inn Fields, London WC2A 3PN

\author{
Received for publication 30 April 1982
}

\begin{abstract}
Summary The relationship between delayed hypersensitivity and granulomatous hypersensitivity to host resistance in mycobacterial infections has been discussed. The grounds for a dissociation between allergic reactions and resistance to infections is reviewed firstly in tuberculosis and then in leprosy. Evidence from clinical observations is compared with data from animal experiments, particularly in mice infected with Mycobacterium lepraemurium. Further comparison is made of the use of sonicated bacterial extracts versus autoclaved whole organisms. The relevance of this discussion to the assessment of the efficacy of the various 'leprosy vaccines', now being tested, is considered.
\end{abstract}

\section{Introduction}

Since the development of tuberculin ${ }^{1}$ and its introduction as a skin testing reagent, there has been considerable controversy as to the role of allergic or hypersensitivity reactions in acquired resistance in mycobacterial infections. Thirty years ago this was reviewed extensively by Rich, ${ }^{2}$ who identified the earliest work on this subject. ${ }^{3}$ In this study it was shown that hypersensitivity produced markedly destructive effects in the tissues and this was related to the level of organisms present. The hypothesis was therefore put forward that acquired resistance in tuberculosis is effected by the accelerated inflammatory reaction caused by local hypersensitivity. The basis of this view was that inflammation is a known protective mechanism in bacterial infections. Inflammation can wall off and prevent the spread of bacteria. In addition, inflammatory exudates are usually strongly bactericidal. Hypersensitivity reactions are among the strongest causes of local inflammation. This view was strengthened by the statement that hypersensitivity as shown by the tuberculin reaction parallels immunity so closely that they must represent 
manifestations of the same phenomenon. Experimentally, guinea-pigs infected with $M$. tuberculosis develop acquired resistance and tuberculin sensitivity in parallel. Neither can be transferred passively with serum, but both can be transferred with lymphoid cell suspensions. Both are therefore manifestations of cell-mediated immunity produced by T-lymphocyte-antigen interaction resulting in the release of lymphokines and macrophage activation. This mechanism of resistance is important as mycobacteria are facultative intracellular parasites capable of multiplying within macrophages, as are other bacteria such as Listeria and Brucella and ce

This view was questioned, ${ }^{2}$ re-enunciated ${ }^{4}$ and questioned again ${ }^{5}$ in the case of tuberculosis. However, it is now important to question the subject again as this view has arisen once more in connection with current work on the production of a leprosy vaccine. It is important that the evidence for parallelism between skin hypersensitivity and host resistance be studied as closely in this infection as in tuberculosis.

\section{Delayed hypersensitivity and cell-mediated immunity in tuberculosis}

Delayed hypersensitivity reactions in the skin are erythematous indurated reactions reaching maximum intensity $24-72 \mathrm{~h}$ after contact with antigenic material. Two types of delayed hypersensitivity reactions may be observed; the tuberculin-type reaction and the Jones-Mote type of reaction. In the tuberculin reaction the skin reaction persists for up to $96 \mathrm{~h}$, and is highly indurated. The Jones-Mote reaction may be observed maximally only $24 \mathrm{~h}$ after skin test. Jones-Mote reactions are highly regulated by suppressor cells and are characterized by large numbers of basophils in a subepidermal position as a result of which they are often referred to as cutaneous basophil hypersensitivity $(\mathrm{CBH})$ reactions. Both these reactions have been shown to be T-lymphocyte mediated reactions. A number of other phenomena have been shown to be similarly $\mathrm{T}$-cell mediated. These include contact sensitivity, skin allograft rejection, organ specific autoimmune processes, tumour immunity, granuloma formation and cellular immunity. Two in vitro tests for cellmediated immunity were introduced. These are the lymphocyte transformation test (LTT) and the leucocyte migration inhibition test (LMIT). All these phenomena have been grouped under the heading cell-mediated immunity (CMI). It therefore appeared natural to consider that they measure the same biological reaction. Numerous attempts have been made to correlate delayed hypersensitivity reactions, LTT and LMIT, both with each other and resistance to infection. One such study in the field of tuberculosis ${ }^{6}$ defines three types of skin test reactions to tuberculin: (a) typical Mantoux reaction; (b) JonesMote hypersensitivity; (c) mixed reaction.

Patients with localized lesions and a prompt response to chemotherapy 
showed typical Mantoux reactions and consistently positive results in the LMIT. Patients with chronic disease with surrounding fibrosis showed JonesMote or mixed reactions and negative LMIT. Patients with rapidly disseminating lesions showed absent skin reactions and LMIT.

However it is stated ${ }^{2}$ that a high degree of hypersensitivity may be associated either with rapidly progressive tuberculosis or with lesions that are being successfully resisted: and a low degree of hypersensitivity is compatible either with lesions that are being well resisted or with devastating ones.

The dissociation between allergy and immunity in tuberculosis in man has also been highlighted in the results of the Medical Research Council trial of tuberculosis vaccine. ${ }^{7}$ In this study there was no correlation between tuberculin sensitivity and protection from tuberculosis in subjects immunized with BCG vaccine. Moreover, one of the sub-strains of the vole bacillus $M$. microti conferred good protection against tuberculosis and poor levels of post-vaccination tuberculin sensitivity.

In a classical study in the guinea-pig, Rothschild et al. ${ }^{8}$ in Rich's laboratory, showed that immunized hypersensitive guinea-pigs could be desensitized with tuberculin so that they no longer reacted hypersensitively with accelerated and exaggerated inflammation and necrosis to the local injection of large amounts of virulent tubercle bacilli or tuberculin. These desensitized guineapigs remained as highly resistant to the proliferation and invasion of the bacilli as were the normal immunized hypersensitive controls. These studies were confirmed in a large number of centres in subsequent years. ${ }^{2}$ The converse was shown ${ }^{9}$ by injecting guinea-pigs with tuberculoprotein and Wax D, so that they became highly tuberculin sensitive but showed no increased resistance to infection. Similar experiments were performed ${ }^{10}$ which produced tuberculin sensitivity in guinea-pigs with an extract of BCG. This hypersensitivity was not accompanied by any increase in resistance when the animals were challenged aerogenically with small numbers of virulent tubercle bacilli. A further dissociation between resistance to infection and hypersensitivity in tuberculosis ${ }^{11}$ was demonstrated in inbred guinea-pigs. It was shown that the strain which showed the most marked hypersensitivity reactions following infection was that which showed the least resistance. In a study ${ }^{12}$ of the resistance of inbred rabbits the conclusion that tuberculin reactivity bore no relation to resistance was reached.

Despite the demonstration, in man, that allergic reactions may not result in immunity, there is the observation that immunity and delayed hypersensitivity develop at the same time in experimental animals. This was shown convincingly for tuberculosis in rabbits ${ }^{13}$ and for Listeria infections in mice, ${ }^{14}$ and led to the view that immunity to tuberculosis was just another manifestation of tuberculin hypersensitivity in which one stable immunogen was involved that was present in killed as well as viable attenuated mycobacteria. It was implied, moreover, that viable mycobacteria immunized better than 
dead organisms because of their ability to multiply in vivo. This produced more antigen and a more sustained immune response. This view, which held considerable attention, was challenged in a series of studies by Youmans and Youmans. ${ }^{15}$ These workers were able to prepare a highly immunogenic fraction from attenuated $M$. tuberculosis H37Ra by disrupting the cells carefully in the cold. Fractionation of the material revealed that the antigen which would confer protection to mice was in a preparation that contained RNA precipitated by ethanol from a ribosomal fraction. No DNA or polysaccharide could be detected but there were significant amounts of protein. Despite producing immunity, neither the bacterial ribosomal fraction nor the RNA produced tuberculin hypersensitivity. Thus immunity to tuberculous infection and tuberculin hypersensitivity would appear to be separate responses of the host to different components of the bacterial cell. Both are mediated by a specific T-lymphocyte response and probably involve macrophage activation. However, as different antigens are involved, the responses can be dissociated although more frequently they run in parallel. It is likely that the granulomatous response is a manifestation of the response to the antigen which produces the allergic reaction, rather than being associated with the basic mechanism involved in host resistance to infection.

\section{Leprosy and Related Animal Models}

Various preparations have been used to assess skin reactivity in man to $M$. leprae. The most frequently used is a preparation of autoclaved whole organism - Mitsuda lepromin. Another reagent commonly used is Dharmendra lepromin in which the organisms are treated with chloroform and ether to remove lipids. A third is a sonicated preparation known as leprosin. Lepromin reactions occur in two phases; there is an early delayed hypersensitivity reaction read at 24 and $48 \mathrm{~h}$ and a later nodular reaction read at 3 weeks. Leprosin reactions are generally read at $48 \mathrm{~h}$. All these reagents may be derived from human skin containing $M$. leprae or from infected armadillo tissue. Reagents are best standardized by a bacillary count of the starting material and reagents containing material from $10^{7}$ organisms $/ \mathrm{ml}$ give the best results for the Dharmendra antigen. ${ }^{16,17}$ The presence of intact bacilli in the reagent, whether Mitsuda or Dharmendra, is necessary for the development of the late nodular component. Soluble or ultrasonicated reagents produced enhanced 24-48 $\mathrm{h}$ reactions and poor late 3 -week Mitsuda reactions. It has been found that the late 3 -week nodular reaction was the best correlate of the clinical status of the individual tested. Patients with lepromatous leprosy (LL) in which there is a specific defect in host resistance to $M$. leprae are inevitably Mitsuda negative, that is they are unable to manifest 3-week nodular reactions, whereas patients at the tuberculoid pole (TT-polar tuberculoid or BT borderline 
tuberculoid) who show a high host resistance to the organism are usually Mitsuda positive. The histology of the 3-week Mitsuda reaction is that of a typical epithelioid cell granuloma, very similar to the lesions in the skin found in TT and BT leprosy. In addition to the lepromin test, cell-mediated immunity may be assessed by the lymphocyte transformation test (LTT) and the leucocyte migration inhibition test (LMIT). Impaired host resistance to M. leprae at the lepromatous pole is associated with a failure of cell-mediated immune response in the LTT and LMIT to specific M. leprae antigens as well as a negative Mitsuda reaction.

It has been found ${ }^{18}$ that circulating lymphocytes from patients with lepromatous leprosy failed to be transformed in vitro by whole $M$. leprae in the LTT. There was a similar failure of M. leprae to produce a positive LMIT. This defect decreased across the leprosy spectrum. Patients at the tuberculoid end showed strong reactivity in both these tests. Although LL lymphocytes could not be transformed by $M$. leprae in many cases they could be transformed by BCG. Although this indicated a strong correlation between the LTT with specific antigen and host resistance, it has been suggested ${ }^{19}$ that the correlation was not necessarily with the ability of the host to eliminate the infecting organism, but with the strength of the allergic reaction shown by the patient. LTT tests were frequently stronger in actively inflamed BT than in TT. In patients with BT leprosy with silent skin lesions there were fairly low LTT responses. Similarly, BL patients with inflamed lesions will react quite strongly in the LTT, and the response may be stronger than in BT patients in whom there is no evidence of inflammation. These observations indicated that the LTT response was related to the state of hypersensitivity of the patient rather than to his resistance to infection. Moreover, the response in the lymphocyte transformation test may vary depending on the nature of the antigenic preparation. ${ }^{20}$ Borderline patients with active nerve damage may show a stronger response using sonicated antigen rather than whole bacilli. Patients with predominantly cutaneous lesions, however, react better with whole bacilli than with sonicated preparations.

It is extremely difficult to compare allergic reactions with host resistance to $M$. leprae in experimental models. The limited growth of $M$. leprae in conventional experimental animals precludes such an approach despite the fact that $M$. leprae induces a strong state of delayed hypersensitivity in guinea-pigs. However, comparison of infection with $M$. lepraemurium in high resistance and low resistance strains of mice provides a useful model for comparing such reactions. ${ }^{21}$ In these studies $\mathrm{C} 57 \mathrm{Bl}$ and $\mathrm{BALB} / \mathrm{c}$ mice were infected subcutaneously with $M$. lepraemurium. C 57 Bl mice are a high resistance in that they are able to limit local multiplication of the organism, whereas $\mathrm{BALB} / \mathrm{c}$ mice are a low resistance strain in which there is rapid multiplication of the organism and centrifugal spread to the draining lymph node. In spite of this difference in local immunity the delayed allergic response induced in 
the contralateral footpad and the in vitro proliferative response of draining lymph node cells to sonicated organisms, were similar in the two strains of mice.

The footpad injection of autoclaved whole $M$. lepraemurium into infected $\mathrm{C} 57 \mathrm{Bl}$ mice gave a prolonged footpad reaction lasting for 4 weeks. ${ }^{22}$ This reaction, which could not be elicited in infected $\mathrm{BALB} / \mathrm{c}$ mice, was considered to be analogous to the Mitsuda reaction in man and showed infiltration with cells of the mononuclear-phagocyte series as well as lymphocytes.

$\mathrm{C} 57 \mathrm{Bl}$ and $\mathrm{BALB} / \mathrm{c}$ mice were also infected with $\mathrm{BCG}$ vaccine. Both strains were equally resistant to the organisms. ${ }^{23}$ Footpad testing with sonicated BCG produced a Jones-Mote type of reaction (transient at $24 \mathrm{~h}$ ) in $\mathrm{BALB} / \mathrm{c}$ mice and a tuberculin type of reaction (persisting up to $72 \mathrm{~h}$ and beyond) in $\mathrm{C} 57 \mathrm{Bl}$ mice during the first 21 days after infection. There was no relationship between the antigen specific lymphocyte transformation test using draining lymph node cells and the footpad reaction. It would also appear that the nature of the delayed hypersensitivity test bore little relation to host resistance in this model.

Certain conclusions can therefore be drawn from these studies. Firstly, that delayed hypersensitivity reactions to sonicated mycobacterial preparations may bear little relation to the lymphocyte response to specific antigen in vitro. Secondly, these reactions do not appear to be a good monitor of host resistance to mycobacteria during the development of infection in inbred strains of mice. A Mitsuda-type of reaction to autoclaved whole mycobacteria would appear to be a better test and shows strong parallelism with host resistance. However, even this is only a true measure of granulomatous hypersensitivity and there is no evidence that it is a real reflection of host resistance.

\section{Skin reactions and in vitro Tests to Assess the Efficacy of Leprosy Vaccines}

There are a number of preparations that have been or are about to be tested for use as a leprosy vaccine. These include the use of Cobalt irradiated or autoclaved $M$. leprae with or without the addition of BCG vaccine ${ }^{24}$ (and in IMMLEP trials), the ICRC bacillus from Bombay ${ }^{25}$ and Mycobacterium W from Delhi (P. Talwar, personal communication). A full trial of a vaccine for increasing host resistance against $M$. leprae in a particular population takes many years to perform. There is therefore pressure to use these vaccines for immunotherapy in lepromatous patients, particularly those with DDS resistance. It is tempting to look for a simple skin test or an in vitro test that would indicate that an individual has regained host resistance. One should therefore ask whether the development of a positive allergic skin test reaction is a true monitor of increased host resistance. There is no doubt that positive delayed hypersensitivity or lymphocyte transformation tests with sonicated 
mycobacteria are no more than reflections of allergic reactivity and need bear no relation to host resistance as has been shown in the mouse models. The main question is what is the significance of a positive granulomatous reaction of the Mitsuda type. There is no doubt again that this is a true allergic reaction, no different from the type of epithelioid cell granuloma produced by metals such as beryllium and zirconium, where there is no question of a relation to host resistance. The animal model shows a strong link with high host resistance, but can a positive granulomatous hypersensitivity response exist in the absence of an increase in host resistance? In the clinical situation can one find cases of a movement across the hypersensitivity spectrum of leprosy towards the tuberculoid pole while the patient remains highly bacilliferous? Can one have a reversal reaction without an increase in host resistance? A rare clinical picture of Lazarine leprosy is described ${ }^{26}$ in which borderline patients develop a tuberculoid clinical picture, but remain a pronounced bacillary population. In this reaction the prognosis as far as nerve involvement is concerned, is poor, as many nerve trunks may be involved and pronounced paralysis may supervene. Thus, there is a risk that the development of a positive Mitsuda reaction in vaccinated leprosy patients might herald the onset of a far more severe neuritis than that seen in the reversal reactions following sulphone therapy.

\section{References}

${ }^{1}$ Koch R. Weitere Mittheilungen über ein Heilmittel gegen Tuberkulose. Deutsch med Wschr, 1890; 16: 1029-32.

${ }^{2}$ Rich AR. The Pathogenesis of Tuberculosis. Second Edition. Blackwell Scientific Publications, Oxford, 1951.

${ }^{3}$ Römer PH. Spezifische Überempfindlichkeit und Tuberkulöseimmunität. Beitr z Klin $d$ Tuberk, 1908; 11: 79-142.

4 Mackaness GB, Blanden RV. Cellular immunity. Progr Allergy, 1967; 11: 89-140.

5 Youmans GP. Relation between delayed hypersensitivity and immunity in tuberculosis. Am Rev Resp Dis, 1975; 111: 109-118.

6 Lenzini L, Rotolli P, Rotolli L. The spectrum of human tuberculosis. Clin exp Immunol, 1977; 27: 230-7.

${ }^{7}$ Hart PD, Sutherland I, Thomas J. The immunity conferred by effective BCG and vole bacillus vaccines in relation to individual variations in induced tuberculin sensitivity and to technical variations in the vaccines. Tubercle, Lond, 1967; 48: 201-10.

${ }^{8}$ Rothschild H, Friedenwald JS, Bernstein C. The relation of allergy to immunity in tuberculosis. Bull Johns Hopkins Hosp, 1934; 54: 232-76.

9 Raffel S. The components of the tubercle bacillus responsible for the delayed type of 'infectious allergy', $J$ infect Dis, 1948; 82: 267-93.

10 Reggiardo Z, Middlebrook G. Delayed type hypersensitivity and immunity against aerogenic tuberculosis in guinea pigs. Infect Immunity, 1974; 9: 815-20.

11 Lewis PA, Loomis D. Ulcerative types as determined by inheritance and as related to natural resistance against tuberculosis. $J$ exp Med, 1928; 47: 449-68. 
12 Lurie MB. Nature of inherited natural resistance to tuberculosis. Proc Soc exp Biol Med, 1938; 39: $181-7$.

13 Lurie MB. Resistance to tuberculosis: experimental studies in native and acquired defensive mechanisms. Harvard University Press, Cambridge, Mass. 1964.

14 Mackaness GB. Cellular resistance to infection. J exp Med, 1962; 116: 381-406.

15 Youmans GP, Youmans AS. Recent studies in acquired immunity in tuberculosis. Current topics in Microbiology and Immunology, 1969; 48: 129-78.

16 Sengupta U, Ramu G, Desikan K V. Assessment of Dharmendra antigen. Leprosy in India, 1978; 50: 599-609.

17 Sengupta U, Ramu G, Desikan KV. Assessment of Dharmendra antigen. II. Standardisation of antigen. Leprosy in India , 1979; 51: 316-22.

18 Myrvang B, Godal T, Ridley DS, Froland SS, Song YK. Immune responsiveness to Mycobacterium leprae and other mycobacterial antigens through the clinical and histological spectrum of leprosy. Clin exp Immunol, 1973; 14: 541-53.

19 Bjune G, Barnetson RS, Ridley DS, Kronvall G. Lymphocyte transformation test in leprosy: correlation of the response with inflammation of lesions. Clin exp Immunol, 1976; 25: 85-94.

Barnetson RS, Bjune G, Pearson JMH, Kronvall G. Antigenic heterogeneity in patients with reactions in borderline leprosy. Br med $J, 1975 ; 4$ : 435-7.

${ }^{21}$ Curtis J, Adu HO, Turk JL. A lack of correlation between antigen specific cellular reactions and resistance to infection to Mycobacterium lepraemurium infection in mice. Immunology, 1981;43: 293-301.

Curtis J, Turk JL. Mitsuda-type lepromin reactions as a measure of host resistance in Mycobacterium lepraemurium infection. Infect Immunity, 1979; 24: 492-500.

${ }^{23}$ Adu HO, Curtis J, Turk JL. Differences in cell-mediated immune responses of 'high resistance' and 'low resistance' mice to a non-pathogenic mycobacterium. Scand $J$ Immunol, 1981; 14: 467-80.

Convit J, Aranzazu N, Pinardi M, Ulrich M. Immunological changes observed in indeterminate and lepromatous leprosy patients and Mitsuda negative contacts after the inoculation of a mixture of Mycobacterium leprae and BCG. Clin exp Immunol, 1979; 36: 214-20.

25 Deo MG, Bapat CV, Chullawalla RG, Bhakti WS. Potential anti-leprosy vaccine from killed ICRC bacilli - A clinicopathological study. Ind J Med Res, 1981; 74: 164-77.

26 Dharmendra. Leprosy, Vol. 1, 1979. Kothari Medical Publishing House, Bombay. 